\title{
THE STELLAR POPULATIONS IN THE DISK OF M31
}

\author{
P.W.MORRIS, W.GRIFFITHS, I.N. REID AND A.J. PENNY, \\ R.J. DICKENS \\ Department of Physics, Leeds University, Leeds, UK; Caltech, \\ USA;Rutherford Appleton Laboratory, UK.
}

CCD observations have been carried on 4 fields in the outer disk and one in the halo of M31 on the 5m Hale telescope, and reduced using the Starman stellar photometry package (Penny, 1992). CMDs for all fields show the brightest 3 magnitudes of the Red Giant Branch. AGB luminosity functions were constructed for each field and corrected for incompletness. Full details are given in Morris et al (1994).

1) The distance modulus of M31 is found to be $(m-M)_{0}=24.4 \pm 0.1$. This was found using the tip of the RGB as a distance indicator.

2) The AGB luminosity function shows very little variation between the fields observed, suggesting that the star formation histories for each field are very similar. The age of the youngest AGB stars is $\sim 5 \mathrm{Gyr}$.

3) The mean colours suggest a metallicity above $[\mathrm{Fe} / \mathrm{H}] \sim-0.8$. All fields show a large colour spread, which may reflect abundance or mass ranges.

4) The disk warp (Innanen etal 1982) seems to be present in the stars of the NE disk as well as $\mathrm{H}$ gas. The stars in the spur field seem to be detached (in space) from the main disk of M31.

5) In this study the intermediate age population of the halo field seems to be almost identical to that of the outer disk - this suggests that the disk and halo were both still under going star formation about $5 \mathrm{Gyr}$ ago and that the stars were being formed out of similar material. This also suggests that the disk and halo were still forming after the formation of the halo globular clusters.

\section{References}

Innanen, K.A, Kamper, K.W., Papp, K.A., and van den Bergh, S., 1982, ApJ, 254, 515 Morris, P.W., Reid, I.N., Griffiths, W.K., and Penny, A.J., 1994, MNRAS, accepted Penny, A.J., Starman A stellar photometry/Image and Table handling package, Starlink Project, Starlink User Note 141.1 